九州大学学術情報リポジトリ

Kyushu University Institutional Repository

\title{
ON THE CONDITIONAL LINEAR STRUCTURAL EQUATIONS WITH LATENT VARIABLES
}

Ke, Hui-xin

Graduate School of Engineering Sciences, and Research Institute of Fundamental Information Science, Kyushu University

Asano, Chooichiro

Research Institute of Fundamental Information Science, Kyushu University

https://doi.org/10.5109/13404

出版情報: Bulletin of informatics and cybernetics. 23 (3/4), pp.171-181，1989-03. Research Association of Statistical Sciences

バージョン :

権利関係 : 


\title{
ON THE CONDITIONAL LINEAR STRUCTURAL EQUATIONS WITH LATENT VARIABLES
}

\author{
By
}

\author{
Hui-xin $\mathrm{KE}^{*}$ and Chooichiro ASANo**
}

\begin{abstract}
This paper discusses the detailed models of partial, bipartial and part linear structural equation, basing on the conditional variancecovariance matrices, and gives concrete algorithms to estimate parameters by the maximum likelihood method and the generalized least square method. An illustration is provided to show how to approach the models.
\end{abstract}

\section{Introduction}

The analysis of structural equation models is well known to be very useful in assessing multivariate data in the fields of social and behavioral sciences. Recently, various models and their analytical methods were discussed and developed, e. g., [8, 9, $17,3,6]$. Among them, Bentler-Weeks model $[3,4]$ was introduced to be a simple general model and has a wide range of applications.

In studying linear relationships among variables, not only the simple correlations or covariances are often needed, but also the conditional correlations or covariances among variables are important to examine, when the effects of some variables are partialled out. In statistical history, partial and multiple correlations were introduced by Yule and then the concepts of part correlation and bipartial correlation were developed by Ezekiel and so on. Roy and latter Rao generalized the concept of canonical correlation first developed by Hotelling to partial canonical correlation, which was further extended to part and bipartial canonical correlations by Timm and Carlson [18] and to $g_{1^{-}}$and $g_{2}$-bipartial canonical correlation by Lee [12]. Recently, Lee [14] considered the analysis of conditional covariance structure models, and proposed the concepts of partial factor analysis and the partial LISREL model.

In the analysis of structural equation model, or generally, the Bentler-Weeks model, the situation is also often faced with eliminating some variables. Therefore we develop in this paper the concepts of partial, bipartial and part linear structural equation models to control the influence of some variables by the appropriate conditional distributions. Thus the estimation of parameters and the goodness-of-fit test can be dealt with analogically as the ordinary case.

* Graduate School of Engineering Sciences, and Research Institute of Fundamental Information Science, Kyushu University, Japan.

** Research Institute of Fundamental Information Sc:ence, Kyushu University 33, Fukuoka 812, Japan. 


\section{The Partial Linear Structural Equation Model}

Using the similar notations as Bentler and Weeks (1980), i. e. $\boldsymbol{\eta}=\boldsymbol{\beta}_{0} \boldsymbol{\eta}+\boldsymbol{\gamma} \boldsymbol{\xi}$ for the structural equation model, $\boldsymbol{Y}=\boldsymbol{\mu}_{y}+\boldsymbol{G}_{y} \boldsymbol{\eta}$ and $\boldsymbol{X}=\boldsymbol{\mu}_{x}+\boldsymbol{G}_{x} \boldsymbol{\xi}$ for the selection model, and $\boldsymbol{\Sigma}_{y y}=\boldsymbol{G}_{y} \boldsymbol{\beta}^{-1} \boldsymbol{\gamma} \boldsymbol{\Phi} \boldsymbol{\gamma}^{\prime} \boldsymbol{\beta}^{\prime-1} \boldsymbol{G}_{y}^{\prime}, \boldsymbol{\Sigma}_{y x}=\boldsymbol{G}_{y} \boldsymbol{\beta}^{-1} \boldsymbol{\gamma} \boldsymbol{\Phi} \boldsymbol{G}_{x}^{\prime}$ and $\boldsymbol{\Sigma}_{x x}=\boldsymbol{G}_{x} \boldsymbol{\phi} \boldsymbol{G}_{x}^{\prime}$ for the complete model, we assume that the vector $\left(\boldsymbol{Y}^{\prime}, \boldsymbol{X}^{\prime}\right)^{\prime}$ belongs to a multivariate normal distribution with mean vector $\boldsymbol{\mu}$ and variance-covariance matrix $\boldsymbol{\Sigma}$.

Thus we shall provide three types of partial models.

(1) Let $\boldsymbol{Y}=\left(\boldsymbol{Y}_{1}^{\prime}, \boldsymbol{Y}_{2}^{\prime}\right)^{\prime}$, where $\boldsymbol{Y}_{1}$ is a $p_{1} \times 1$ random vector and $\boldsymbol{Y}_{2}$ is a $p_{2} \times 1$ random vector, $\left(p_{1}+p_{2}=p\right)$. Suppose $\boldsymbol{Y}_{1}$ and $\boldsymbol{Y}_{2}$ satisfy the following selection model,

$$
\boldsymbol{Y}=\left(\begin{array}{l}
\boldsymbol{Y}_{1} \\
\boldsymbol{Y}_{2}
\end{array}\right)=\boldsymbol{\mu}_{y}+\boldsymbol{G}_{y} \boldsymbol{\eta}=\left(\begin{array}{l}
\boldsymbol{\mu}_{y_{1}} \\
\boldsymbol{\mu}_{y_{2}}
\end{array}\right)+\left(\begin{array}{c}
\boldsymbol{G}_{y_{1}} \\
\boldsymbol{G}_{y_{2}}
\end{array}\right) \boldsymbol{\beta}^{-1} \boldsymbol{\gamma} \boldsymbol{\xi}
$$

where $\boldsymbol{G}_{y_{1}}$ and $\boldsymbol{G}_{y_{2}}$ are known $p_{1} \times m$ and $p_{2} \times m$ selection matrices, and $\boldsymbol{\mu}_{y_{1}}$ and $\boldsymbol{\mu}_{y_{2}}$ are $p_{1} \times 1$ and $p_{2} \times 1$ mean vectors, respectively. Hence the population variance-covariance matrix of $Y$ can be written as

$$
\boldsymbol{\Sigma}_{y y}=\mathrm{C}\left(\boldsymbol{Y}, \boldsymbol{Y}^{\prime}\right)=\left(\begin{array}{c}
\boldsymbol{\Sigma}_{y_{1} y_{1}} \boldsymbol{\Sigma}_{y_{1} y_{2}} \\
\boldsymbol{\Sigma}_{y_{2} y_{1}} \boldsymbol{\Sigma}_{y_{2} y_{2}}
\end{array}\right)=\left(\begin{array}{c}
\boldsymbol{G}_{y_{1}} \\
\boldsymbol{G}_{y_{2}}
\end{array}\right) \boldsymbol{\beta}^{-1} \boldsymbol{\gamma} \boldsymbol{\Phi} \boldsymbol{\gamma}^{\prime} \boldsymbol{\beta}^{\prime-1}\left(\boldsymbol{G}_{y_{1}}^{\prime} \boldsymbol{G}_{y_{2}}^{\prime}\right),
$$

where $\mathrm{C}\left(\boldsymbol{Y}, \boldsymbol{Y}^{\prime}\right)$ expresses the variance-covariance matrix of $\boldsymbol{Y}$.

We are interested in the structure of the model after elimination of the influence of the variables $\boldsymbol{Y}_{2}$. Consider the conditional variance-covariance matrix of $\left(\boldsymbol{Y}_{1}^{\prime}, \boldsymbol{X}^{\prime}\right)^{\prime}$, when $\boldsymbol{Y}_{2}=\boldsymbol{y}_{2}$ is given. Using the well known formula

we have

$$
\Sigma_{11.2}=\Sigma_{11}-\Sigma_{12} \Sigma_{22}^{-1} \Sigma_{21}
$$

where

$$
\mathrm{C}\left(\left(\begin{array}{l}
\boldsymbol{Y}_{1} \\
\boldsymbol{X}
\end{array}\right),\left.\left(\begin{array}{l}
\boldsymbol{Y}_{1} \\
\boldsymbol{X}
\end{array}\right)^{\prime}\right|_{y_{2}}\right)=\left(\begin{array}{c}
\boldsymbol{\Sigma}_{y_{1} y_{1} \cdot y_{2}} \boldsymbol{\Sigma}_{y_{1} x \cdot y_{2}} \\
\boldsymbol{\Sigma}_{x y_{1} \cdot y_{2}} \boldsymbol{\Sigma}_{x x \cdot y_{2}}
\end{array}\right),
$$

$$
\begin{aligned}
& \boldsymbol{\Sigma}_{y_{1} y_{1} \cdot y_{2}}=G_{y_{1}} \boldsymbol{\beta}^{-1} \boldsymbol{\gamma} \Phi \boldsymbol{\gamma}^{\prime} \boldsymbol{\beta}^{\prime-1} G_{y_{1}}^{\prime}-G_{y_{1}} \boldsymbol{\beta}^{-1} \boldsymbol{\gamma} \Phi \boldsymbol{\gamma}^{\prime} \boldsymbol{\beta}^{\prime-1} \boldsymbol{G}_{y_{2}}^{\prime} \boldsymbol{\Sigma}_{y_{2} y_{2}}^{-1} \boldsymbol{G}_{y_{2}} \boldsymbol{\beta}^{-1} \boldsymbol{\gamma} \Phi \boldsymbol{\gamma}^{\prime} \boldsymbol{\beta}^{\prime-1} \boldsymbol{G}_{y_{1}}^{\prime} \\
& =G_{y_{1}} \beta^{-1} \boldsymbol{\gamma}\left(\Phi-\Phi \boldsymbol{\gamma}^{\prime} \boldsymbol{\beta}^{\prime-1} G_{y_{2}}^{\prime} \Sigma_{y_{2} y_{2}}^{-1} G_{y_{2}} \boldsymbol{\beta}^{-1} \boldsymbol{\gamma} \Phi\right) \boldsymbol{\gamma}^{\prime} \boldsymbol{\beta}^{\prime-1} G_{y_{1}}^{\prime} \text {, } \\
& \boldsymbol{\Sigma}_{y_{1} x y_{2}}=G_{y_{1}} \boldsymbol{\beta}^{-1} \boldsymbol{\gamma}\left(\boldsymbol{\Phi}-\boldsymbol{\Phi} \boldsymbol{\gamma}^{\prime} \boldsymbol{\beta}^{\prime-1} G_{y_{2}}^{\prime} \boldsymbol{\Sigma}_{y_{2} y_{2}}^{-1} \boldsymbol{G}_{y_{2}} \boldsymbol{\beta}^{-1} \boldsymbol{\gamma} \boldsymbol{\Phi}\right) \boldsymbol{G}_{x}^{\prime} \text {, } \\
& \boldsymbol{\Sigma}_{x x \cdot y_{2}}=\boldsymbol{G}_{x}\left(\boldsymbol{\Phi}-\boldsymbol{\Phi} \boldsymbol{\gamma}^{\prime} \boldsymbol{\beta}^{\prime-1} \boldsymbol{G}_{y_{2}}^{\prime} \boldsymbol{\Sigma}_{y_{2} y_{2}}^{-1} \boldsymbol{G}_{y_{2}} \boldsymbol{\beta}^{-1} \boldsymbol{\gamma} \boldsymbol{\Phi}\right) \boldsymbol{G}_{x}^{\prime} \text {. }
\end{aligned}
$$

Consider the variance-covariance matrix of $\boldsymbol{\xi}$ and $\boldsymbol{Y}_{2}$, then we have

$$
\mathrm{C}\left(\left(\begin{array}{c}
\boldsymbol{\xi} \\
\boldsymbol{Y}_{2}
\end{array}\right),\left(\begin{array}{c}
\boldsymbol{\xi} \\
\boldsymbol{Y}_{2}
\end{array}\right)^{\prime}\right)=\left(\begin{array}{cc}
\boldsymbol{\Phi} & \boldsymbol{\Phi}_{\boldsymbol{\gamma}^{\prime} \boldsymbol{\beta}^{\prime-1} \boldsymbol{G}_{y_{2}}^{\prime}}
\end{array}\right) .
$$

Thus the conditional variance-covariance matrix of $\boldsymbol{\xi} \mid \boldsymbol{y}_{2}$ can be obtained as

$$
\mathrm{C}\left(\boldsymbol{\xi}, \boldsymbol{\xi}^{\prime} \mid \boldsymbol{y}_{2}\right)=\boldsymbol{\Phi}-\boldsymbol{\Phi} \boldsymbol{\gamma}^{\prime} \boldsymbol{\beta}^{\prime-1} G_{y_{2}}^{\prime} \boldsymbol{\Sigma}_{y_{2} y_{2}}^{-1} \boldsymbol{G}_{y_{2}} \boldsymbol{\beta}^{-1} \boldsymbol{\gamma} \boldsymbol{\Phi}=\boldsymbol{\Phi}_{2} \text {. }
$$

This means that the elements in $\boldsymbol{\Phi}_{2}$ give the partial covariances among variables in $\boldsymbol{\xi}$ with $\boldsymbol{Y}_{2}$ held constant. Therefore we can rewrite the conditional model in the following way. Let 


$$
\boldsymbol{Y}^{\sharp}=\boldsymbol{Y}_{1}\left|\boldsymbol{y}_{2}, \quad \boldsymbol{X}^{\ddagger}=\boldsymbol{X}\right| \boldsymbol{y}_{2}, \quad \eta^{\sharp}=\eta\left|\boldsymbol{y}_{2}, \quad \boldsymbol{\xi}^{\ddagger}=\boldsymbol{\xi}\right| \boldsymbol{y}_{2},
$$

then

$$
\begin{aligned}
& \eta^{=}=\boldsymbol{\beta}_{0} \eta^{\#}+\gamma \boldsymbol{\xi}^{*}, \quad \boldsymbol{Y}^{\#}=\mu_{y_{1}}+\boldsymbol{G}_{y_{1}} \eta^{\#}, \quad \boldsymbol{X}^{\ddagger}=\boldsymbol{\mu}_{x}+\boldsymbol{G}_{x} \boldsymbol{\xi}^{\#}, \\
& \boldsymbol{\Sigma}_{y \neq y}==G_{y_{1}} \boldsymbol{\beta}^{-1} \boldsymbol{\gamma} \Phi_{2} \boldsymbol{\gamma}^{\prime} \boldsymbol{\beta}^{-1} G_{y_{1}}^{\prime}, \quad \boldsymbol{\Sigma}_{y} x_{x} \neq=G_{y_{1}} \boldsymbol{\beta}^{-1} \boldsymbol{\gamma} \Phi_{2} G_{x}^{\prime}, \\
& \boldsymbol{\Sigma}_{x \ddagger x}==\boldsymbol{G}_{x} \boldsymbol{\Phi}_{2} \boldsymbol{G}_{x}^{\prime},
\end{aligned}
$$

which are of the same form as the ordinary Bentler-Weeks model. We may call (2.6) and (2.7) the type-1 partial linear structural equation model with $\boldsymbol{Y}_{2}$ partialled out. Thus, after $\boldsymbol{Y}_{2}$ being controlled, $\boldsymbol{\eta}\left|\boldsymbol{y}_{2}, \boldsymbol{\xi}\right| \boldsymbol{y}_{2}, \boldsymbol{Y}_{1} \mid \boldsymbol{y}_{2}$ and $\boldsymbol{X} \mid \boldsymbol{y}_{2}$ still construct a Bentler-Weeks model.

(2) Let

$$
\boldsymbol{X}=\left(\begin{array}{l}
\boldsymbol{X}_{1} \\
\boldsymbol{X}_{2}
\end{array}\right)=\boldsymbol{\mu}_{x}+\boldsymbol{G}_{x} \boldsymbol{\xi}=\left(\begin{array}{l}
\boldsymbol{\mu}_{x_{1}} \\
\boldsymbol{\mu}_{x_{2}}
\end{array}\right)+\left(\begin{array}{l}
\boldsymbol{G}_{x_{1}} \\
\boldsymbol{G}_{x_{2}}
\end{array}\right) \boldsymbol{\xi}
$$

where $\boldsymbol{X}_{1}$ and $\boldsymbol{X}_{2}$ are $q_{1} \times 1$ and $q_{2} \times 1\left(q_{1}+q_{2}=q\right)$ random vectors, $\boldsymbol{\mu}_{x_{1}}$ and $\boldsymbol{\mu}_{x_{2}}$ are $q_{1} \times 1$ and $q_{2} \times 1$ mean vectors, and $\boldsymbol{G}_{x_{1}}$ and $\boldsymbol{G}_{x_{2}}$ are $q_{1} \times n$ and $q_{2} \times n$ selection matrices, respectively. By the similar discussion, we then have the following type- 2 partial linear structural equation model with the effects of the variables $\boldsymbol{X}_{2}$ being controlled.

$$
\begin{aligned}
& Y^{\ddagger}=\boldsymbol{Y}\left|\boldsymbol{x}_{2}, \quad \boldsymbol{X}^{\ddagger}=\boldsymbol{X}_{1}\right| \boldsymbol{x}_{2}, \quad \eta^{\neq}=\eta\left|\boldsymbol{x}_{2}, \quad \xi^{\ddagger}=\xi\right| \boldsymbol{x}_{2}, \\
& \eta^{*}=\beta_{0} \eta^{*}+\gamma \xi^{*}, \quad Y \neq=\mu_{y}+G_{y} \eta^{*}, \quad X=\mu_{x_{1}}+G_{x_{1}} \xi^{\#},
\end{aligned}
$$

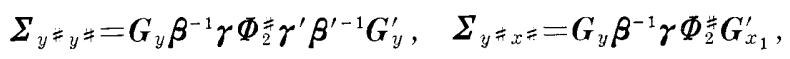

$$
\begin{aligned}
& \boldsymbol{\Sigma}_{x \neq x} \approx=\boldsymbol{G}_{x_{1}} \Phi \boldsymbol{\Phi}_{x_{1}}^{\prime},
\end{aligned}
$$

where $\Phi_{2}^{\ddagger}=\Phi-\Phi G_{x_{2}}^{\prime} \Sigma_{x_{2} x_{2}}^{-1} \boldsymbol{G}_{x_{2}} \boldsymbol{\Phi}$ is the conditional variance-covariance matrix of $\boldsymbol{\xi}$ given $\boldsymbol{X}_{2}$, that is $\boldsymbol{\xi} \mid \boldsymbol{x}_{2}$. Hence, the type-2 partial model is also a Bentler-Weeks model.

(3) In the third type, we have both partitioned random vectors

$$
\boldsymbol{Y}=\left(\boldsymbol{Y}_{1}^{\prime}, \boldsymbol{Y}_{2}^{\prime}\right)^{\prime} \text { and } \boldsymbol{X}=\left(\boldsymbol{X}_{1}^{\prime}, \boldsymbol{X}_{2}^{\prime}\right)^{\prime}
$$

as showed in (2.1) and (2.8) and consider the situation, where the effects of both $\boldsymbol{Y}_{2}$ and $\boldsymbol{X}_{2}$ have been controlled. Let

$$
\begin{aligned}
& \boldsymbol{Y}==\boldsymbol{Y}_{1}\left|\left(y_{2}, \boldsymbol{x}_{2}\right), \quad \boldsymbol{X}^{*}=\boldsymbol{X}_{1}\right|\left(y_{2}, \boldsymbol{x}_{2}\right), \\
& \eta^{\#}=\eta\left|\left(y_{2}, \boldsymbol{x}_{2}\right), \quad \boldsymbol{\xi}^{\ddagger}=\boldsymbol{\xi}\right|\left(y_{2}, \boldsymbol{x}_{2}\right) .
\end{aligned}
$$

This time the conditional variance-covariance matrix of $\boldsymbol{Y}_{1}$ given both $\boldsymbol{y}_{2}$ and $\boldsymbol{x}_{2}$ is as follows

$$
\begin{aligned}
\boldsymbol{\Sigma}_{y^{\sharp} y_{\ddagger}} & =\mathrm{C}\left(\boldsymbol{Y}^{\ddagger}, \boldsymbol{Y}^{\ddagger \prime}\right)=\mathrm{C}\left(\boldsymbol{Y}_{1}, \boldsymbol{Y}_{1}^{\prime} \mid \boldsymbol{y}_{2}, \boldsymbol{x}_{2}\right)=\boldsymbol{\Sigma}_{y_{1} y_{1} \cdot y_{2} x_{2}}=\boldsymbol{\Sigma}_{y_{1} y_{1} \cdot x_{2}}-\boldsymbol{\Sigma}_{y_{1} y_{2} \cdot x_{2}} \boldsymbol{\Sigma}_{y_{2} y_{2} \cdot x_{2}}^{-1} \boldsymbol{\Sigma}_{y_{2} y_{1} \cdot x_{2}} \\
& =\boldsymbol{G}_{y_{1}} \boldsymbol{\beta}^{-1} \boldsymbol{\gamma} \Phi_{2}^{\#} \boldsymbol{\gamma}^{\prime} \boldsymbol{\beta}^{\prime-1} \boldsymbol{G}_{y_{1}}^{\prime}-\boldsymbol{G}_{y_{1}} \boldsymbol{\beta}^{-1} \boldsymbol{\gamma} \boldsymbol{\Phi}_{2}^{\prime} \boldsymbol{\gamma}^{\prime} \boldsymbol{\beta}^{\prime-1} G_{y_{2}}^{\prime} \boldsymbol{\Sigma}_{y_{2} y_{2} \cdot x_{2}}^{-1} \boldsymbol{G}_{y_{2}} \boldsymbol{\beta}^{-1} \boldsymbol{\gamma} \boldsymbol{\Phi}_{2}^{\#} \boldsymbol{\gamma}^{\prime} \boldsymbol{\beta}^{\prime-1} \boldsymbol{G}_{y_{1}}^{\prime} \\
& =\boldsymbol{G}_{y_{1}} \boldsymbol{\beta}^{-1} \boldsymbol{\gamma} \boldsymbol{\Phi}_{22} \boldsymbol{\gamma}^{\prime} \boldsymbol{\beta}^{\prime-1} \boldsymbol{G}_{y_{1}}^{\prime},
\end{aligned}
$$

where $\Phi_{22}=\Phi_{2}^{\ddagger}-\Phi_{2}^{\ddagger} \boldsymbol{\gamma}^{\prime} \boldsymbol{\beta}^{\prime-1} G_{y_{2}}^{\prime} \boldsymbol{\Sigma}_{y_{2} y_{2} \cdot x_{2}}^{-1} \boldsymbol{G}_{y_{2}} \boldsymbol{\beta}^{-1} \boldsymbol{\gamma} \Phi_{2}^{\ddagger}$, and $\boldsymbol{\Phi}_{22}$ is the conditional variancecovariance matrix of $\boldsymbol{\xi}$ given both $\boldsymbol{y}_{2}$ and $\boldsymbol{x}_{2}$. That is

$$
\mathrm{C}\left(\boldsymbol{\xi}^{*}, \boldsymbol{\xi}^{\ddagger \prime}\right)=\mathrm{C}\left(\boldsymbol{\xi}, \boldsymbol{\xi}^{\prime} \mid \boldsymbol{y}_{2}, \boldsymbol{x}_{2}\right)=\Phi_{22} .
$$


Similarly, we can obtain

Let

$$
\begin{aligned}
& \boldsymbol{\Sigma}_{y=x}==\mathrm{C}\left(\boldsymbol{Y}_{1}, \boldsymbol{X}_{1}^{\prime} \mid \boldsymbol{y}_{2}, \boldsymbol{x}_{2}\right)=\boldsymbol{G}_{y_{1}} \boldsymbol{\beta}^{-1} \boldsymbol{\gamma} \Phi_{2_{2}} G_{x_{1}}^{\prime}, \\
& \boldsymbol{\Sigma}_{x} x_{x} \#=\mathrm{C}\left(\boldsymbol{X}_{1}, \boldsymbol{X}_{1}^{\prime} \mid \boldsymbol{y}_{2}, \boldsymbol{x}_{2}\right)=\boldsymbol{G}_{x_{1}} \boldsymbol{\Phi}_{22} \boldsymbol{G}_{x_{\mathrm{i}}}^{\prime} \text {. }
\end{aligned}
$$

$$
Y^{\#}=\mu_{y_{1}}+G_{y_{1}} \eta^{\#}, \quad X^{\#}=\mu_{x_{1}}+G_{x_{1}} \xi^{*} .
$$

In this case, (2.11), (2.12), (2.14) and (2.15) form the type-3 partial linear structural equation model, we call, which is also a Bentler-Weeks model with both $\boldsymbol{I}_{2}$ and $\boldsymbol{X}_{2}$ partialled out.

\section{The Bipartial Linear Structural Equation Model}

Let us now consider a structure of the model after controlling both effects of $\boldsymbol{Y}_{2}$ on $Y_{1}$ and of $\boldsymbol{X}_{2}$ on $\boldsymbol{X}_{1}$. This leads us to the bipartial linear structural equation model. Consider the following conditional random vectors

$$
\boldsymbol{Y}^{\sharp}=\boldsymbol{Y}_{1}\left|\boldsymbol{y}_{2}, \quad \boldsymbol{X}^{\#}=\boldsymbol{X}_{1}\right| \boldsymbol{x}_{2} .
$$

The corresponding variance and covariance matrices can be expressed as

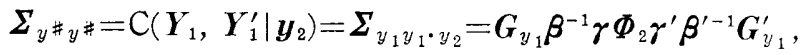

$$
\begin{aligned}
& \Sigma_{x \# \text { \#\# }}=\mathrm{C}\left(X_{1}, X_{1}^{\prime} \mid x_{2}\right)=\Sigma_{x_{1} x_{1} \cdot x_{2}}=G_{x_{1}} \Phi_{2}^{\sharp} G_{x_{1}}^{\prime} \text {. }
\end{aligned}
$$

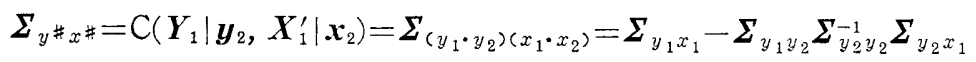

$$
\begin{aligned}
& -\boldsymbol{\Sigma}_{y_{1} x_{2}} \boldsymbol{\Sigma}_{x_{2} x_{2}}^{-1} \boldsymbol{\Sigma}_{x_{2} x_{1}}+\boldsymbol{\Sigma}_{y_{1} y_{2}} \boldsymbol{\Sigma}_{y_{2} y_{2}}^{-1} \boldsymbol{\Sigma}_{y_{2} x_{2}} \boldsymbol{\Sigma}_{x_{2} x_{2}}^{-1} \boldsymbol{\Sigma}_{x_{2} x_{1}}=G_{y_{1}} \beta^{-1} \boldsymbol{\gamma} \boldsymbol{\Phi} G_{x_{1}}^{\prime} \\
& -G_{y_{1}} \beta^{-1} \boldsymbol{\gamma} \Phi \boldsymbol{\gamma}^{\prime} \boldsymbol{\beta}^{\prime-1} G_{y_{2}}^{\prime} \boldsymbol{\Sigma}_{y_{2} y_{2}}^{-1} G_{y_{2}} \beta^{-1} \boldsymbol{\gamma} \Phi G_{x_{1}}^{\prime}-G_{y_{1}} \beta^{-1} \boldsymbol{\gamma} \Phi G_{x_{2}}^{\prime} \Sigma_{x_{2} x_{2}}^{-1} G_{x_{2}} \Phi G_{x_{1}}^{\prime} \\
& +G_{y_{1}} \boldsymbol{\beta}^{-1} \boldsymbol{\gamma} \Phi \boldsymbol{\gamma}^{\prime} \boldsymbol{\beta}^{\prime-1} G_{y_{2}}^{\prime} \boldsymbol{\Sigma}_{y_{2} y_{2}}^{-1} G_{y_{2}} \boldsymbol{\beta}^{-1} \boldsymbol{\gamma} \Phi G_{x_{2}}^{\prime} \boldsymbol{\Sigma}_{x_{2} x_{2}}^{-1} G_{x_{2}} \Phi G_{x_{1}}^{\prime} \\
& =G_{y_{1}} \beta^{-1} \boldsymbol{\gamma}\left(\Phi_{2} \Phi^{-1} \Phi_{2}^{\#}\right) G_{x_{1}}^{\prime} .
\end{aligned}
$$

Let $\boldsymbol{\Phi}_{22}^{\#}=\boldsymbol{\Phi}_{2} \boldsymbol{\Phi}^{-1} \boldsymbol{\Phi}_{2}^{\#}$, and let two quasi random vectors be $\boldsymbol{\xi}^{\sharp}=\boldsymbol{\xi} \mid \boldsymbol{y}_{2}$ and $\boldsymbol{\xi}^{\sharp \neq=}=\boldsymbol{\xi} \mid \boldsymbol{x}_{2}$. Then we have

Let

$$
\mathrm{C}\left(\boldsymbol{\xi}^{\#}, \boldsymbol{\xi}^{\# \prime}\right)=\Phi_{2}, \quad \mathrm{C}\left(\boldsymbol{\xi}^{\# \#}, \boldsymbol{\xi}^{\# \# \prime}\right)=\Phi_{2}^{\#}, \quad \mathrm{C}\left(\boldsymbol{\xi}^{\#}, \boldsymbol{\xi}^{* \prime \prime}\right)=\Phi_{22}^{*} .
$$

Then we have

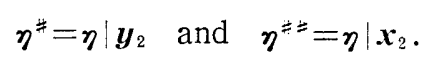

$$
\begin{aligned}
& \eta^{\#}=\beta_{0} \eta^{\sharp}+\gamma \xi^{\#}, \quad \eta^{\# \#}=\beta_{0} \eta^{\# \#}+\gamma \xi^{\# \#}, \\
& Y^{\#}=\mu_{y_{1}}+G_{y_{1}} \eta^{\#}, \quad X^{\#}=\mu_{x_{1}}+G_{x_{1}} \xi^{\# \#},
\end{aligned}
$$

and

$$
\begin{aligned}
& \boldsymbol{\Sigma}_{y \# y}=\boldsymbol{G}_{y_{1}} \boldsymbol{\beta}^{-1} \boldsymbol{\gamma} \Phi_{2} \boldsymbol{\gamma}^{\prime} \boldsymbol{\beta}^{\prime-1} G_{y_{1}}^{\prime}, \\
& \boldsymbol{\Sigma}_{y \sharp x}==G_{y_{1}} \boldsymbol{\beta}^{-1} \boldsymbol{\gamma} \Phi_{22}^{\#} G_{x_{1}}^{\prime}, \\
& \boldsymbol{\Sigma}_{x \sharp x}, \\
& =G_{x_{1}} \boldsymbol{\Phi}_{2}^{\ddagger} G_{x_{1}}^{\prime} .
\end{aligned}
$$

This is a bipartial linear structural equation model, and is a quasi Bentler-Weeks model, with elimination of both the effects of $\boldsymbol{Y}_{2}$ on $\boldsymbol{Y}_{1}$ and of $\boldsymbol{X}_{2}$ on $\boldsymbol{X}_{1}$. 


\section{The Part Linear Structural Equation Model}

For Bentler-Weeks model, a "complete" latent variable model is considered. However, when the problem is to analyze some kind of longitudinal data, in which the same quantitative measurements are obtained at two or more occasions and all the measured variables are assumed to be functions of some latent variables, we may have two sets of observed data $\boldsymbol{Y}^{(1)}$ and $\boldsymbol{Y}^{(2)}$ but without $\boldsymbol{X}$ variables. In these two sets, sometimes there may be nuisance variables. In such situation, it leads us to a part linear structural equation model by eliminating the effects of them.

Assume that longitudinal data $\boldsymbol{Y}^{(1)}$ and $\boldsymbol{Y}^{(2)}$ can be expressed by the partitioned form

$$
\boldsymbol{Y}=\left(\begin{array}{c}
\boldsymbol{Y}_{1} \\
\boldsymbol{Y}_{2} \\
\boldsymbol{Y}_{3} \\
\boldsymbol{Y}_{4}
\end{array}\right)=\left(\begin{array}{c}
\boldsymbol{\mu}_{1} \\
\boldsymbol{\mu}_{2} \\
\boldsymbol{\mu}_{3} \\
\boldsymbol{\mu}_{4}
\end{array}\right)+\left(\begin{array}{l}
G_{1} \\
G_{2} \\
G_{3} \\
\boldsymbol{G}_{4}
\end{array}\right) \eta,
$$

where $\boldsymbol{Y}_{2}$ and $\boldsymbol{Y}_{4}$ are the respective $p_{2} \times 1$ and $p_{4} \times 1$ nuisance variables in $\boldsymbol{Y}^{(1)}$ and $\boldsymbol{Y}^{(2)}$, and are distinguished from $\boldsymbol{Y}_{1}\left(p_{1} \times 1\right)$ and $\boldsymbol{Y}_{3}\left(p_{3} \times 1\right)$, i. e. $p_{1}+p_{2}+p_{3}+p_{4}=p, \boldsymbol{\mu}_{i}$ and $\boldsymbol{G}_{i}$ have the same meanings as the former sections for $i=1,2,3,4$.

Thus consider the conditional random vector

$$
Y^{*}=\left(\begin{array}{c}
Y_{1} \mid y_{2} \\
Y_{3} \mid y_{4}
\end{array}\right)
$$

Then

where

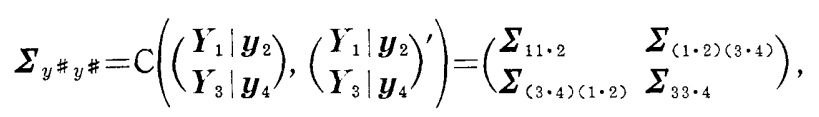

and

$$
\begin{aligned}
& \Sigma_{11 \cdot 2}=G_{1} \beta^{-1} \boldsymbol{\gamma} \Phi_{2} \boldsymbol{\gamma}^{\prime} \boldsymbol{\beta}^{\prime-1} G_{1}^{\prime}, \\
& \Sigma_{33 \cdot 4}=G_{3} \beta^{-1} \boldsymbol{\gamma} \Phi_{4} \boldsymbol{\gamma}^{\prime} \boldsymbol{\beta}^{\prime-1} G_{3}^{\prime}, \\
& \boldsymbol{\Sigma}_{(1 \cdot 2)(3 \cdot 4)}=G_{1} \boldsymbol{\beta}^{-1} \boldsymbol{\gamma} \Phi_{24} \boldsymbol{\gamma}^{\prime} \boldsymbol{\beta}^{\prime-1} G_{3}^{\prime},
\end{aligned}
$$

$$
\begin{aligned}
& \Phi_{2}=\Phi-\Phi \boldsymbol{\gamma}^{\prime} \boldsymbol{\beta}^{\prime-1} G_{2}^{\prime} \Sigma_{22}^{-1} G_{2} \boldsymbol{\beta}^{-1} \boldsymbol{\gamma} \Phi=\mathrm{C}\left(\boldsymbol{\xi}, \boldsymbol{\xi}^{\prime} \mid \boldsymbol{y}_{2}\right), \\
& \Phi_{4}=\Phi-\Phi_{\boldsymbol{\gamma}} \boldsymbol{\beta}^{\prime-1} G_{4}^{\prime} \Sigma_{44}^{-1} G_{4} \beta^{-1} \boldsymbol{\gamma} \Phi=\mathrm{C}\left(\boldsymbol{\xi}, \boldsymbol{\xi}^{\prime} \mid \boldsymbol{y}_{4}\right) \text {, } \\
& \Phi_{24}=\Phi_{2} \Phi^{-1} \Phi_{4}=\mathrm{C}\left(\boldsymbol{\xi}\left|\boldsymbol{y}_{2}, \boldsymbol{\xi}^{\prime}\right| \boldsymbol{y}_{4}\right),
\end{aligned}
$$

where $\Phi=\mathrm{C}\left(\boldsymbol{\xi}, \boldsymbol{\xi}^{\prime}\right)$.

For this case, we can define the part linear structural equation model as a kind of quasi Bentler-Weeks model in the following way

where

$$
\begin{aligned}
& \eta^{\#}=B_{0} \eta^{\#}+\Gamma \xi^{\#}, \\
& Y^{\#}=\mu+G \eta^{\#}, \\
& \Sigma^{\#}=\Sigma_{y} y_{y}=G B^{-1} \Gamma \Phi^{\#} \Gamma^{\prime} B^{\prime-1} G^{\prime},
\end{aligned}
$$

$$
\eta^{*}=\left(\begin{array}{l}
\eta \mid y_{2} \\
\eta \mid y_{4}
\end{array}\right), \quad \xi^{*}=\left(\begin{array}{l}
\xi \mid y_{2} \\
\xi \mid y_{4}
\end{array}\right)
$$




$$
\begin{aligned}
& \boldsymbol{B}_{0}=\left(\begin{array}{cc}
\boldsymbol{\beta}_{0} & 0 \\
0 & \boldsymbol{\beta}_{0}
\end{array}\right), \quad \Gamma=\left(\begin{array}{ll}
\boldsymbol{\gamma} & 0 \\
0 & \gamma
\end{array}\right), \\
& \boldsymbol{G}=\left(\begin{array}{cc}
G_{1} & 0 \\
0 & \boldsymbol{G}_{3}
\end{array}\right), \quad \boldsymbol{\mu}=\left(\begin{array}{l}
\boldsymbol{\mu}_{1} \\
\boldsymbol{\mu}_{3}
\end{array}\right), \\
& \boldsymbol{\Phi}^{\#}=\mathrm{C}\left(\boldsymbol{\xi}^{\#}, \boldsymbol{\xi}^{\# \prime}\right)=\left(\begin{array}{ll}
\Phi_{2} & \boldsymbol{\Phi}_{24} \\
\Phi_{42} & \Phi_{4}
\end{array}\right), \\
& \boldsymbol{B}=I-\boldsymbol{B}_{0} .
\end{aligned}
$$

The orders of the matrices in (4.7) are $2 m \times 1,2 n \times 1,2 m \times 2 m, 2 m \times 2 n,\left(p_{1}+p_{3}\right) \times 2 m$, $\left(p_{1}+p_{3}\right) \times 1,2 n \times 2 n$ and $2 m \times 2 m$, respectively.

Note that the concept of "part model" described in this section is somewhat different from Timm and Carlson [18]. While the latter is based on the part correlation coefficient between $\boldsymbol{Y}$ and $\boldsymbol{X}$ after partialling out $\boldsymbol{Z}$ from $\boldsymbol{X}$, but not from $\boldsymbol{Y}$, the former is only a part of the ordinary Bentler-Weeks model without $\boldsymbol{X}$-variables in it.

\section{Estimation of the Models and Test of Goodness-of-fit}

Since the conditional models in above sections are either Bentler-Weeks models or quasi Bentler-Weeks models, the usual methods of estimation and goodness-of-fit test can be used here without difficulty.

Therefore, saving the space, we only discuss the case for the part model and omit the partial and bipartial models.

Suppose that $\boldsymbol{Z}_{1}, \boldsymbol{Z}_{2}, \cdots, \boldsymbol{Z}_{N}$ are a random sample of observations from a population $\mathrm{N}(\boldsymbol{\mu}, \boldsymbol{\Sigma})$, where $\boldsymbol{Z}_{i}=\left(\boldsymbol{Y}_{1_{i}}^{\prime}, \boldsymbol{Y}_{2_{i}}^{\prime}, \boldsymbol{Y}_{3_{i}}^{\prime}, \boldsymbol{Y}_{4_{i}}^{\prime}\right)^{\prime}$ is a $p \times 1$ partitioned vector with four subvectors of dimensions $p_{1}, p_{2}, p_{3}$ and $p_{4}$, respectively. Let $\boldsymbol{S}=\left(\boldsymbol{S}_{i j}\right)$ be the partitioned sample variance-covariance matrix, where $i, j=1,2,3,4$. Let $\Theta$ be a $t \times 1$ vector of unconstrained parameters in $\boldsymbol{\Phi}^{\#}, \boldsymbol{\gamma}$ and $\boldsymbol{\beta}_{0},\left(\boldsymbol{\Theta}_{0}\right.$ is the true vector of $\left.\boldsymbol{\theta}\right)$, and $\boldsymbol{\Sigma}$ be a function of $\theta$. Let

$$
S^{\ddagger}=\left(\begin{array}{ll}
\boldsymbol{S}_{11 \cdot 2} & \boldsymbol{S}_{(1 \cdot 2)(3 \cdot 4)} \\
\boldsymbol{S}_{(3 \cdot 4)(1 \cdot 2)} & \boldsymbol{S}_{33 \cdot 4}
\end{array}\right)
$$

be the conditional sample variance-covariance matrix, where

$$
\begin{aligned}
& S_{11 \cdot 2}=S_{11}-S_{12} S_{22}^{-1} S_{21}, \\
& S_{3 \cdot \cdot 4}=S_{33}-S_{34} S_{44}^{-1} S_{43}, \\
& S_{(1 \cdot 2)(3 \cdot 4)}=S_{13}-S_{12} S_{22}^{-1} S_{23}-S_{14} S_{44}^{-1} S_{43}+S_{12} S_{22}^{-1} S_{24} S_{44}^{-1} S_{43},
\end{aligned}
$$

and let $\Sigma^{\#}$ be the corresponding partitioned conditional variance-covariance matrix of the population. $S^{\#}$ is proved to be an unbiased estimator of $\boldsymbol{\Sigma}^{\#}$ by use of the results from Anderson [1]. The estimator of $\Theta$ is obtained by minimizing an objective function or a fit function which describes the difference between $S^{\#}$ and $\boldsymbol{\Sigma}^{\#}$. According to Browne [5], the G.L.S. estimator $\hat{\boldsymbol{\theta}}$ of $\boldsymbol{\theta}$ results from minimizing

$$
Q(\boldsymbol{\Theta})=\operatorname{tr}\left\{\left[\boldsymbol{S}^{\#}-\boldsymbol{\Sigma}^{\#}(\boldsymbol{\Theta})\right] \boldsymbol{W}\right\}^{2} / 2,
$$

and the M.L. estimator $\tilde{\Theta}$ of $\Theta$ is obtained by minimizing 


$$
F(\boldsymbol{\Theta})=\log \left|\boldsymbol{\Sigma}^{\#}(\boldsymbol{\Theta})\right|+\operatorname{tr}\left\{\boldsymbol{S}^{\#}\left[\boldsymbol{\Sigma}^{\#}(\boldsymbol{\Theta})\right]^{-1}\right\}-\log \left|\boldsymbol{S}^{\#}\right|-\left(p_{1}+p_{3}\right),
$$

where $\boldsymbol{W}$ is a weighted matrix to converge asymptotically to a positive definite matrix, and usually $\boldsymbol{W}$ is chosen as $\boldsymbol{W}=\boldsymbol{S}^{\#-1}$. The algorithms may be Gauss-Newton algorithm and Scoring algorithm [15]. Clearly speaking, the basic steps in Gauss-Newton algorithm for minimizing $Q(\boldsymbol{\Theta})$ and in Scoring algorithm for minimizing $F(\boldsymbol{\Theta})$ are

and

$$
\boldsymbol{\Theta}_{k+1}=\boldsymbol{\Theta}_{k}-\alpha_{k} \boldsymbol{H}_{k}^{-1} \boldsymbol{g}_{k}, \quad\left(0 \leqq \alpha_{k} \leqq 1\right)
$$

$$
\boldsymbol{\Theta}_{k+1}=\Theta_{k}-\alpha_{k} \boldsymbol{I}_{k}^{-1} \boldsymbol{q}_{k}, \quad\left(0 \leqq \alpha_{k} \leqq 1\right)
$$

respectively, where

$$
\begin{aligned}
& \boldsymbol{H}_{k}=\left(\partial \boldsymbol{\Sigma}^{\#} / \partial \Theta\right)(\boldsymbol{W} \otimes \boldsymbol{W})\left(\partial \boldsymbol{\Sigma}^{\#} / \partial \boldsymbol{\theta}\right)^{\prime}, \\
& \boldsymbol{g}_{k}=\left(\partial \boldsymbol{\Sigma}^{\#} / \partial \boldsymbol{\theta}\right)(\boldsymbol{W} \otimes \boldsymbol{W}) \operatorname{vec}\left(\boldsymbol{\Sigma}^{\#}-\boldsymbol{S}^{\#}\right), \\
& \boldsymbol{I}_{k}=\left(\partial \boldsymbol{\Sigma}^{\#} / \partial \Theta\right)\left(\boldsymbol{\Sigma}^{\#-1} \otimes \boldsymbol{\Sigma}^{\#-1}\right)\left(\partial \boldsymbol{\Sigma}^{\#} / \partial \Theta\right)^{\prime}, \\
& \boldsymbol{q}_{k}=\left(\partial \boldsymbol{\Sigma}^{\#} / \partial \boldsymbol{\partial}\right)\left(\boldsymbol{\Sigma}^{\#-1} \otimes \boldsymbol{\Sigma}^{\#-1}\right) \operatorname{vec}\left(\boldsymbol{\Sigma}^{\#}-\boldsymbol{S}^{\#}\right),
\end{aligned}
$$

and $\operatorname{vec} \boldsymbol{X}$ is a vector consisting of all elements of $\boldsymbol{X}$ taken row by row. For the case when there are functional constraints on parameters, the results are given in $\mathrm{Ke}$ and Asano [10].

The derivative $\partial \Sigma^{\#} / \partial \Theta$ can be worked out first with respect to $\Theta^{\#}$, which is a vector of all elements in $\Phi^{\#}, \boldsymbol{\gamma}$ and $\boldsymbol{\beta}_{0}$, to get

$$
\left(\partial \Sigma^{\#} / \partial \Theta^{\#}\right)^{\prime}=\left[\left(\partial \Sigma^{\#} / \partial \Phi^{\#}\right)^{\prime},\left(\partial \Sigma^{\#} / \partial \gamma\right)^{\prime},\left(\partial \Sigma^{\#} / \partial \beta_{0}\right)^{\prime}\right]
$$

and then the appropriate rows corresponding to constants or linear constraints are eliminated [10].

We can work out the following results

$$
\begin{aligned}
& \partial \Sigma^{\#} / \partial \Phi^{\#}=\left(\boldsymbol{\Gamma}^{\prime} \boldsymbol{B}^{\prime-1} \boldsymbol{G}^{\prime} \otimes \boldsymbol{\Gamma}^{\prime} \boldsymbol{B}^{\prime-1} \boldsymbol{G}^{\prime}\right), \\
& \partial \boldsymbol{\Sigma}^{\sharp} / \partial \gamma=\boldsymbol{D}_{m n}\left(\boldsymbol{B}^{\prime-1} \boldsymbol{G}^{\prime} \otimes \boldsymbol{\Phi} \boldsymbol{\Gamma}^{\prime} \boldsymbol{B}^{\prime-1} \boldsymbol{G}^{\prime}\right)\left(\boldsymbol{I}+\boldsymbol{E}_{q q}\right), \\
& \partial \boldsymbol{\Sigma}^{\ddagger} / \partial \boldsymbol{\beta}_{0}=\boldsymbol{D}_{m n}\left(\boldsymbol{B}^{\prime-1} \boldsymbol{G}^{\prime} \otimes \boldsymbol{B}^{-1} \boldsymbol{\Gamma} \boldsymbol{\Phi} \boldsymbol{\Gamma}^{\prime} \boldsymbol{B}^{\prime-1} \boldsymbol{G}^{\prime}\right)\left(\boldsymbol{I}+\boldsymbol{E}_{q q}\right)
\end{aligned}
$$

where $q=p_{1}+p_{3}$, and $\boldsymbol{E}_{q q}$ denotes $\partial \boldsymbol{X}^{\prime} / \hat{\partial} \boldsymbol{X}$ for matrix $X(q \times q)$ without constants or functionally dependent elements. $\boldsymbol{D}_{m n}=\left(d_{\boldsymbol{g} h}\right)$ is an $m n \times 4 m n$ matrix with typical elements

$$
d_{g h}= \begin{cases}1, & \text { if } h=g+(2 m+k) n, \text { where }(k-1) n+1 \leqq g \leqq k n, 1 \leqq k \leqq m, \\ 0, & \text { otherwise. }\end{cases}
$$

It has been proved and also for the constrained case that the obtained G. L.S. estimator $\hat{\Theta}$ possesses the following asymptotic properties under some imposed regularity conditions, Browne [5] and Lee and Bentler [13],

(1) it is consistent,

(2) it is asymptotically equivalent to the maximum likelihood estimator,

(3) the asymptotic distribution of $\widehat{\Theta}-\boldsymbol{\Theta}$ is $\mathrm{N}\left(\boldsymbol{O}, \boldsymbol{M}_{0}\right)$, where

$$
\boldsymbol{M}_{0}=2 n^{-1}\left[\left(\partial \boldsymbol{\Sigma}_{0}^{\sharp} / \partial \Theta\right)\left(\boldsymbol{\Sigma}_{0}^{\#-1} \otimes \boldsymbol{\Sigma}_{0}^{\#-1}\right)\left(\partial \boldsymbol{\Sigma}_{0}^{\#} / \partial \Theta\right)^{\prime}\right]^{-1},
$$


(4) the asymptotic distribution of $n Q(\hat{\Theta})$ is Chi-square with degrees of freedom $q(q+1) / 2-t$.

The property (3) can be used to obtain the standard errors of estimator $\hat{\theta}$ and the property (4) to test the goodness-of-fit of the proposed model.

\section{A Numerical Illustration}

We shall illustrate the proposed idea and methods by using a part of the longitudinal data, Jöreskog and Sörbom [8]. The data consist of scores in the following six tests recorded in two grades.

$$
\begin{aligned}
& Y_{1}(\mathrm{MATH}): \text { mathematics, } \quad Y_{2}(\mathrm{SCI}): \text { science, } \\
& Y_{3}(\mathrm{SS}): \text { social studies, } \quad Y_{4}(\mathrm{READ}): \text { reading, } \\
& Y_{5}(\mathrm{SCATV}): \text { verbal part of SCAT }(\mathrm{Sch} \text { (SAstic Aptitude Test }), \\
& Y_{6}(\mathrm{SCATQ}): \text { Quantitative part of SCAT, }
\end{aligned}
$$

where the data were analyzed by Lee and Poon [16] with a constrained factor analytic model and by $\mathrm{Ke}$ and Asano [11] with a linear structural equation model.

In this paper, the data are further analyzed on a part linear structural equation model for the first variables (MATH) in two grades held constant, by the proposed algorithm. The solution for the part conditional structural equation model is presented in Table 1, and the results of the unconditional model in Table 2, that is, the data were analyzed by simply deleting the first variables in two grades without any

Table 1. Results for the conditional part model

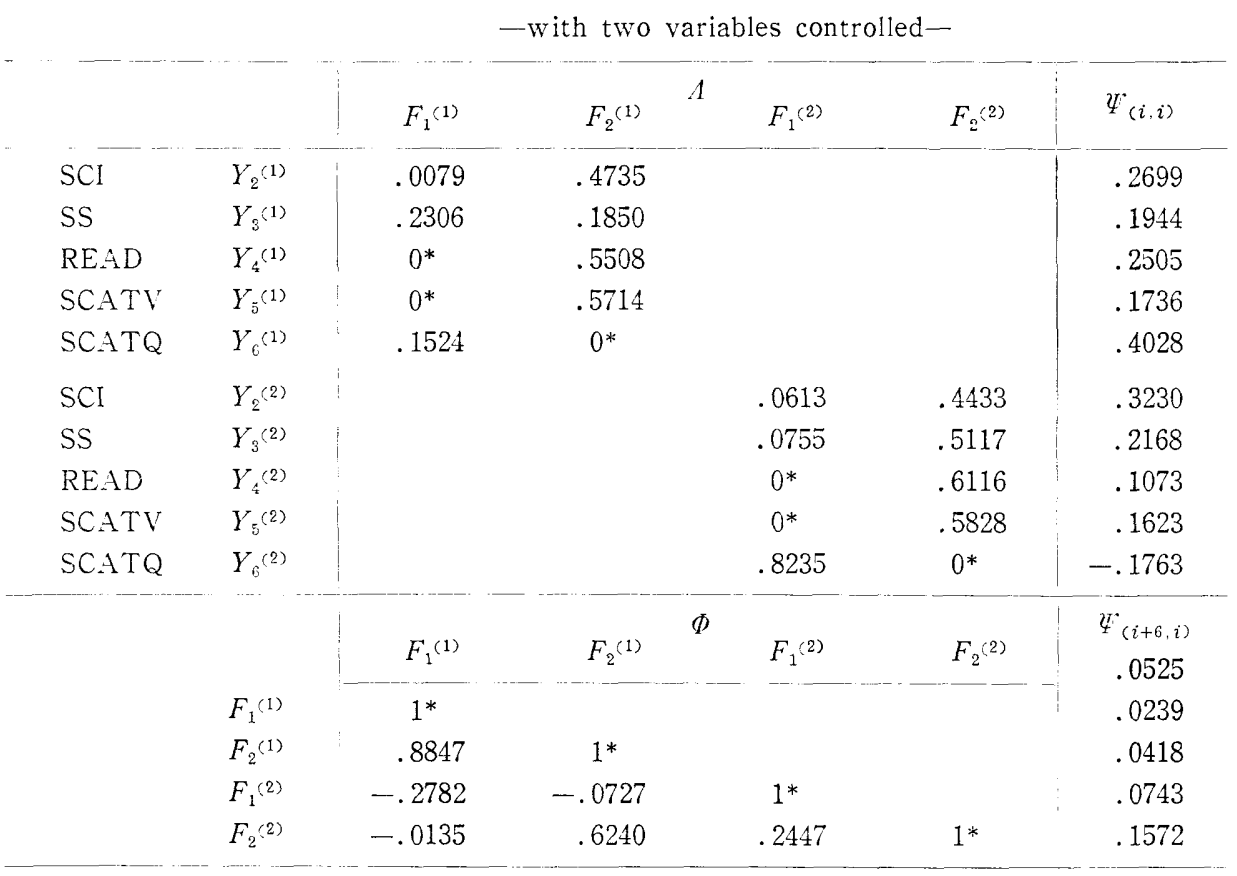

Note: An asterisk indicates that the parameter was fixed at this value 
Table 2. Results for the unconditional model

-with two variables deleted--

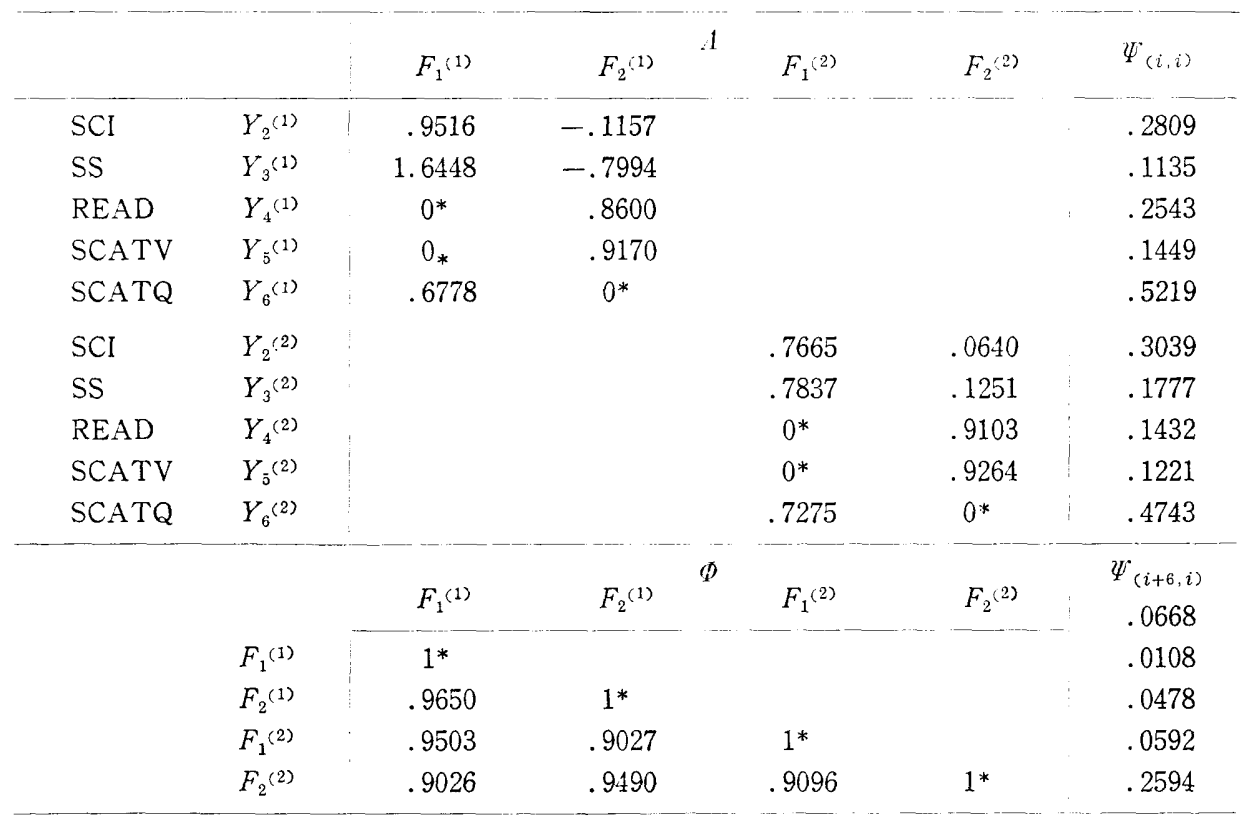

Table 3. Results for the complete model

\begin{tabular}{|c|c|c|c|c|c|c|c|}
\hline & & $F_{1}^{(1)}$ & $F_{2}^{(1)}$ & A & $F_{1}^{(2)}$ & $F_{2}^{(2)}$ & $\Psi_{(i, i)}$ \\
\hline MATH & $Y_{1}^{(1)}$ & .9261 & $0^{*}$ & & & & .1358 \\
\hline $\mathrm{SCI}$ & $Y_{2}^{(1)}$ & .1493 & .6901 & & & & .2728 \\
\hline SS & $Y_{3}^{(1)}$ & .4208 & .4395 & & & & .1509 \\
\hline READ & $Y_{4}^{(1)}$ & $0^{*}$ & .8565 & & & & .2541 \\
\hline SCATV & $Y_{5}(1)$ & $0^{*}$ & .9044 & & & & .1648 \\
\hline SCATQ & $Y_{6}{ }^{(1)}$ & .7902 & $0^{*}$ & & & & .3535 \\
\hline MATH & $Y_{1}^{(2)}$ & & & & .9075 & $0^{*}$ & .1512 \\
\hline $\mathrm{SCI}$ & $Y_{2}^{(2)}$ & & & & .1775 & .6392 & .3104 \\
\hline SS & $Y_{3}{ }^{(2)}$ & & & & .1291 & .7505 & .2053 \\
\hline READ & $Y_{4}^{(2)}$ & & & & $0^{*}$ & .9222 & .1060 \\
\hline SCATV & $Y_{5}(2)$ & & & & $0^{*}$ & .9071 & .1547 \\
\hline \multirow[t]{7}{*}{ SCATQ } & $Y_{6}{ }^{(2)}$ & & & & .7912 & $0^{*}$ & .3538 \\
\hline & & $F_{1}^{(1)}$ & $F_{2}^{(1)}$ & $\Phi$ & $F_{1}^{(2)}$ & $F_{2}{ }^{(2)}$ & $\begin{array}{r}\Psi_{(i+6, i)} \\
-.0558\end{array}$ \\
\hline & & & & & & & .0614 \\
\hline & $F_{1}(1)$ & $1^{*}$ & & & & & .0233 \\
\hline & $F_{2}(1)$ & .8213 & $1^{*}$ & & & & .0394 \\
\hline & $F_{1}^{(2)}$ & .9475 & .7667 & & $1^{*}$ & & .0789 \\
\hline & $F_{2}^{(2)}$ & .8278 & .9584 & & .8354 & $1^{*}$ & .1309 \\
\hline
\end{tabular}

Note: An asterisk indicates that the parameter was fixed at this value 
conditional model.

It will be noted from Table 1 and Table 2 that the results for conditional and unconditional cases are quite different, in view of the factor loadings and factor correlations. In fact, the factors have completely different meanings in these two cases. For the conditional part model (case 1), factors $F_{1}$ and $F_{2}$ still keep the similar meanings as the complete model with six variables in Table 3 computed out by the algorithm in $\mathrm{Ke}$ and Asano [11], that is, $F_{1}$ shows the mathematical and quantitative ability, while $F_{2}$ the reading and verbal ability. It can be understood that after eliminating the effects of MATH, most of the loading coefficients of the first factor $F_{1}$ become very small. But the loading of SCATQ on $F_{1}{ }^{(2)}$ in the second grade gets larger. This may be due to the changes in the tests SCATQ and MATH from the earier to the later grades. "In the earier grade, these tests consist mainly of arithmetic items whereas in the later grade they are made up of items measuring logical reasoning and presented in verbal form" [8]. For the unconditional model (case 2), we have nothing to do with the variable MATH at all. Factor $F_{1}$ may show highly the general knowledge for science and society, and $F_{2}$ the ability for reading and verbalism. Also it will be pointed out that the correlation between two factors in case 2 is rather large, while the correlation in case 1 is not so significant.

\section{References}

[1] Anderson, T.W.: An introduction to multivariate statistical analysis, second edition, John Wiley \& Sons, (1971).

[2] Bentler, P.M.: Multivariate analysis with latent variable: causal modeling, Ann. Rev. of Psychology, 31 (1980), 419-456.

[3] Bentler, P.M. and Weeks, D.G.: Linear structural equations with latent variables, Psychometrika, 45 (1980), 289-308.

[4] Bentler, P.M. and Weeks, D.G.: Multivariate analysis with latent variables, in: KrishNaiah, P.R. and Kanal, L. N. eds., Handbook of statistics, North-Holland Publishing Company, 2 (1982), 747-771.

[5] Browne, M.W.: Generalized least squares estimators in the analysis of covariance structures, South African Statist. J., 8 (1974), 1-24.

[6] Browne, M.W.: Covariance structures. in: Hawkrns, D.M. (ed.), Topics in applied multivariate analysis, Cambridge University Press, London, (1982).

[7] Jöreskog, K.G.: A general method for analysis of covariance structures, Biometrika, 51 (1970), 239-251.

[8] Jöreskog, K.G. and Sörbom, D.: Statistical models and methods for analysis of longitudinal data, In: Aiguer, D. J. and Goldberger, A.S. (eds.), Latent variables in socioeconomic models, North-Holland, Amsterdam, (1977).

[9] Jöreskog, K. G.: Structural analysis of covariance and correlation matrix, Psychometrika, 43 (1978), 443-477.

[10] KE, H.X. and AsANo, Ch.: Estimation of linear structural equation model with arbitrary constraints on parameters, Behaviormetrika, 1 (1988a), 81-89.

[11] KE, H.X. and AsANo, Ch.: An algorithm for linear structural equation model, (1988b), Submitted for publication.

[12] LEE, S. Y.: Generalizations of the partial, part and bipartial canonical correlation analysis, Psychometrika, 43 (1978), 427-431.

[13] LEe, S.Y. and Bentler, P.M.: Some asymptotic properties of constrained generalized least squares estimation in covariance structure models, South African Statist. J., 4 (1980), 121-136. 
[14 LEE, S.Y.: Analysis of conditional covariance structure models, Computational statistics \& data analysis, 4 (1986), 41-59.

[15] LEe, S.Y. and Jenvrich, R.I.: A study of algorithms for covariance structure analysis with specific comparisons using factor analysis, Psychometrika, 44 (1979), 99-113.

[16] LEE, S.Y. and POON, W. Y.: Further developments on constrained estimation in analy'sis of covariance structure, The statistician, 34 (1985), 305-316.

[17] McDonAld, P.R.: A simple comprehensive model for the analysis of covariance structures, British Journal of mathematical and statistical Psychology, 3 (1978), 59-72.

[18- Timm, N.H. and CARLson, J.E.: Part and bipartial canonical correlation analysis, Psychometrika, 41 (1976), 159-176.

Received September 13, 1988 